\title{
Enhancing Provider Reporting of Notifiable Diseases using HIE- enabled Decision Support
}

\author{
Brian E. Dixon 1, 2 , Shaun J. Grannis 4,2 , Joe Gibson 3 \\ ${ }^{1}$ Epidemiology, Indiana University Fairbanks School of Public Health, Indianapolis, Indiana, United States, ${ }^{2}$ Regenstrief Institute, Indianapolis, Indiana, \\ United States, ${ }^{3}$ Marion County Public Health Department, Indianapolis, Indiana, United States, ${ }^{4}$ Indiana University School of Medicine, Indianapolis, \\ Indiana, United States

\section{Objective}

To enhance the process by which outpatient providers report surveillance case information to public health authorities following a laboratory-confirmed diagnosis of a reportable disease.

\section{Introduction}

Traditionally, public health agencies (PHAs) wait for hospital, laboratory or clinic staff to initiate case reports. However, this passive approach is burdensome for reporters and produces incomplete and delayed reports, which can hinder assessment of disease in the community and potentially delay recognition of patterns and outbreaks [1].

Modern surveillance practice is shifting toward greater use of electronically transmitted disease information. The adoption of electronic health record (EHR) systems and health information exchange (HIE) among clinical organizations and systems, driven by policies such as the 'meaningful use' program, is creating an information infrastructure that public health organizations can take advantage of to improve surveillance practice [2].

\section{Methods}

Using an existing HIE infrastructure, we developed, tested and implemented an intervention that pre-populates an electronic version of the official Indiana State Department of Health communicable disease reporting form following detection of a notifiable disease in an electronic laboratory message. Pre-populated form fields included patient demographics, notifiable disease confirmatory test results, and provider information. The intervention was delivered electronically to the provider using the HIE network. Seven representative, high priority diseases were included: chlamydia, gonorrhea, syphilis, salmonella, hepatitis B, hepatitis C, and histoplasmosis.

The intervention was implemented in seven diverse primary care clinics in central Indiana. Control clinics were all other outpatient settings connected to the HIE that reported at least one communicable disease case to the county PHA during the same time period. The primary outcome measure was reporting rate (provider reports received as a percent of all case reports received by the PHA for individuals with a notifiable disease). Clinic-submitted case reports were collected between 9/9/2013 - 3/15/2014 and 9/15/2014 $-6 / 12 / 2016$ from all clinics (intervention and non-intervention) that reported to the county PHA before and during the intervention period. We grouped case reports into non-intervention and intervention groups based on whether or not the laboratory test occurred at an intervention clinic during the clinic's intervention period. Reports received from intervention clinics outside of the clinics' intervention period were classified as non-intervention reports.

To evaluate the impact of the intervention on the outcome measures, we employed a difference-in-difference approach in which the change in reporting rates among intervention clinics before and after implementation was compared to the change in reporting rates among non-intervention clinics during the same timeframes. The analysis utilized generalized linear models with logistic regression using the logit link function for reporting rate. All statistical tests were performed using the NLestimate macro within Enterprise SAS Version 9.4. 


\section{Results}

Of 16,172 unique cases observed across all time periods, 1,453 (9\%) were reported by intervention clinics. During intervention time periods, provider reporting rates significantly increased from $20 \%$ to $50 \%$ in intervention clinics while falling from $12 \%$ to $10 \%$ in the control clinics $(\mathrm{p}<0.001)$.

The most prevalent conditions observed overall were hepatitis B $(5,362 ; 33.5 \%)$, chlamydia $(5,157 ; 32.2 \%)$, and hepatitis C (3,236; $20.2 \%$ ). Only chlamydia, gonorrhea, and hepatitis C possessed enough observations during the intervention periods for robust comparisons.

With respect to the change in reporting rates for specific diseases, rates increased for chlamydia (56.9\% vs. $66.4 \%)$, gonorrhea (55.6\% vs. $58.3 \%)$, and Hepatitis C $(6.5 \%$ vs. $7.3 \%)$ in the intervention clinics. However, only the increase for chlamydia was statistically significant $(\mathrm{p}<0.001)$. Rates for all three diseases decreased in control clinics during the same time period.

\section{Conclusions}

The results of the study indicate that electronic, pre-populated case reporting forms integrated into providers' EHR systems and workflow, enabled by an interoperable HIE network, can be effective at increasing clinic reporting rates. These results are encouraging and offer hope for initiatives that aim to help PHAs leverage the expanding health IT infrastructure created through policies like 'meaningful use.'

\section{Acknowledgement}

This project was supported by grant number R01HS020909 from the Agency for Healthcare Research and Quality. The content is solely the responsibility of the authors and does not necessarily represent the official views of the Agency for Healthcare Research and Quality.

\section{References}

1. Revere D, Hills RH, Dixon BE, Gibson PJ, Grannis SJ. 2017. Notifiable condition reporting practices: implications for public health agency participation in a health information exchange. BMC Public Health. 17(1), 247. PubMed https://doi.org/10.1186/s12889-017-4156-4

2. Dixon B, Grannis S. Public Health Informatics Infrastructure. In: Magnuson JA, Fu JPC, editors. Public Health Informatics and Information Systems. Health Informatics: Springer London; 2014. p. 69-88. 